\title{
Editorial: "Novel Pain Therapeutics: From Basic Research to Clinical Translation and Rehabilitation"
}

\author{
Damiana Scuteri ${ }^{1,2}$, Tsukasa Sakurada $^{3}$, Paolo Tonin $^{2}$, Maria Tiziana Corasaniti ${ }^{4,5}$ and \\ Giacinto Bagetta ${ }^{1 *}$
}

${ }^{1}$ Pharmacotechnology Documentation and Transfer Unit, Section of Preclinical and Translational Pharmacology, Department of Pharmacy, Health and Nutritional Sciences, University of Calabria, Rende, Italy, ${ }^{2}$ Regional Center for Serious Brain Injuries, S. Anna Institute, Crotone, Italy, ${ }^{3}$ Center for Supporting Pharmaceutical Education, Faculty of Pharmaceutical Sciences, Daiichi University of Pharmacy, Fukuoka, Japan, ${ }^{4}$ Department of Health Sciences, University "Magna Graecia” of Catanzaro, Catanzaro, Italy, ${ }^{5}$ School of Hospital Pharmacy, University "Magna Graecia" of Catanzaro, Catanzaro, Italy

Keywords: pain, sensitization, natural and synthetic analgesics, opioids, dementia, cognitive impairment, rehabilitation

Editorial on the Research Topic

Novel Pain Therapeutics: From Basic Research to Clinical Translation and Rehabilitation

Chronic pain is a major public health problem representing a global leading cause of disability and disease burden with low back pain and headache disorders ranking top-ten as causes of disabilityadjusted life-years (DALYs) throughout the whole lifetime from teenage to old age (Diseases and Injuries, 2020). In fact, some $30-50 \%$ of the world population and almost $20 \%$ of the Europeans actually suffer from chronic pain with devastating cost for the society in terms of disability and hospitalization (Breivik et al., 2006; Reid et al., 2011; van Hecke et al., 2013; Mills et al., 2019). Therefore, research aiming at understanding the mechanisms involved in chronic pain is the key to improve treatment and quality of life of patients. This is even more important at the time of COVID19 that poses several limitations to the access to chronic pain treatment (Karos et al., 2020), enhancing its impact especially on aged, cognitively impaired and non communicative patients (Scuteri et al., 2020b). For a better understanding and management of pain there are four main aspects to be considered: 1) neurotransmission and anomalous plasticity; 2) neuron-glial interaction in central sensitization; 3) drugs and botanicals for pain therapy; 4) pharmacology, assessment and management of chronic pain in the fragile populations. Nociceptive primary afferent fibers release mainly glutamate, substance $\mathrm{P}$ and calcitonin gene-related peptide (CGRP); modulation of pain by these neurotransmitters is fundamental for translation into clinical practice. Accordingly, understanding of the role of CGRP neurotransmission represents a noteworthy example of advances in migraine prevention and treatment, previously relying only on triptans and symptomatic, non specific, analgesics (Scuteri et al., 2020a). The progress guaranteed by antiCGRP monoclonal antibodies for migraine, and by eptinezumab, in particular, allowing faster action due to intravenous administration (Scuteri et al., 2019b), is due to the dissection of CGRP signaling mechanisms (Scuteri et al., 2019a). The modulation of the endogenous pain inhibitory system is implicated in the action of pain-relieving drugs (Ossipov et al., 2010). Metabotropic glutamate receptors can promote chronic pain through sensitization of the central nucleus of the amygdala (Ossipov et al., 2010). Thus, glutamatergic transmission is involved in the ascending but also in the descending pathways modulation due to presynaptic glutamate metabotropic autoreceptors like mGlu2/3. The latter are involved in the mechanisms of antinociception of drugs and botanicals (Scuteri et al., 2019d). In fact, the essential oil of bergamot is able to modulate synaptic level of 
glutamate (Morrone et al., 2007) and this contributes to its analgesic activity (Scuteri et al., 2019c), which is enriched by anxiolytic properties devoid of sedation (Rombola et al., 2019). Interestingly, the analgesic effect occurs also when it is administered via the inhalatory route (Scuteri et al., 2018a), though it is independent on olfactory stimulation and mediated by systemic absorption of active principles (Hamamura et al.). Trazodone has been demonstrated to restore acutely the aberrant plasticity to which mGlu autoreceptors are subjected after spinal nerve ligation (Cisani et al.). Furthermore, glutamate is involved in the antinociceptive effect of apelin, the endogenous ligand for the putative receptor protein related to the type 1 angiotensin receptor (APJ) orphan G-protein-coupled receptor (Lv et al.). The activation of $\mathrm{N}$-methyl-D-aspartate (NMDA) receptor elicits cholecystokinin-8 (CCK-8) pronociceptive effects (Hayashi et al.). Transient Receptor Potential (TRP) Vanilloid 1 and Ankyrin 1 (TRPV1, TRPA1) channels represent important targets for analgesia at level of the nociceptive primary sensory neurons (Horváth et al.). The neuropeptide oxytocin is endowed with preemptive analgesic properties in a validated postoperative pain model (Espinosa De Los Monteros-Zuniga et al.). Galanin receptor agonists have been demonstrated to have potential analgesic properties with action on protein kinase C ( $\mathrm{Li}$ et al.). Opioid receptors occurring on nociceptor terminals play a pivotal role in pain modulation. For instance, in neuropathic pain deltorphin II can inhibit the response of $\mathrm{C}$ fibers in a concentration-dependent and delta opioid receptors-mediated manner (Joukal et al.). Moreover, methadone has been found to be effective in morphine-resistant hyperalgesia and to restore morphine antinociception in the inflammatory model of the complete Freund's adjuvant (CFA) (Watanabe et al.). The latter is interesting also in view of the lack of efficacy and the onset of serious side effects for chronic administration of opioids in the treatment of osteoarthritis. The role of the inflammasome in pain is fundamental and the interaction between immune and nervous system contributes to the development of chronic pain. Also, the modulation of microglia can reduce allodynia in a sexdependent manner (Saika et al.). Oxidative stress and nuclear factor erythroid derived-2-related factor 2 (Nrf2) pathway is notably implicated in neuropathic pain (Zhao et al.). Other targets for pain treatment are the small-conductance $\mathrm{Ca}^{2+}$ activated $\mathrm{K}^{+}$channels for visceral hypersensitivity (Ji et al.). In particular, the study of visceral hypersensitivity is of the utmost importance since it can predispose to visceral chronic pain and it can be caused by maternal separation responsible for elevated firing frequency of corticotropin-releasing factor neurons (Huang et al.). Among the non pharmacologic approaches, acupuncture has been studied in primary dysmenorrhea and its efficacy can be predicted by painrelated functional connectivity patterns through machine learning multivariate pattern analysis ( $\mathrm{Yu}$ et al.); the endocannabinoid system seems to be implicated in electroacupuncture-induced analgesia (MacDonald and Chen). Hence, the use of novel technological tools, e.g., machine learning, can improve the comprehension of the machinery of chronic pain. A better understanding of the above mechanisms can lead to discover the properties of already known and widely used analgesics, like acetaminophen, which still holds some surprises, e.g., the action of its metabolite $\mathrm{N}$-acylphenolamine on brain TRPV1 and cannabinoid 1 receptors with consequent central analgesia (Ohashi and Kohno). These findings highlight the need for more basic as well as clinical studies on commonly used painkillers. One of the most remarkable is the case of opioids, often inappropriately used in fragile populations of the elderly commonly suffering from diseases that impair their communication skills. The treatment of chronic pain per se represents an area of strong therapeutic inappropriateness resulting in real-world limited access to care for fragile patients, e.g., demented aged people (Scuteri et al., 2017; Scuteri et al., 2018b; Scuteri et al., 2021). This is mirrored by the scarce use of the existing adequate observational pain scales and by the lack of standardization and guidelines for clinical practice in dementia (Gimenez-Llort et al.). This issue is very serious also for patients suffering from post-stroke pain. In fact, what emerges from the systematic review and meta-analysis of current literature is that the use of opioids in these patients is not specifically studied in the latter population with clinical trials designed for this purpose and using suitable pain assessment tools (Scuteri et al.). The assessment and the treatment of pain after spinal cord injury is still an unmet need (Finnerup, 2013; Yasko and Mains, 2018). The correct management of pain in patients who have been subjected to a severe brain injury with disorders of consciousness is fundamental to allow the best therapeutic approach and the planning of the most effective rehabilitation strategy (Riganello et al.).

\section{DATA AVAILABILITY STATEMENT}

All the datasets generated/analyzed for this study are included in the article.

\section{AUTHOR CONTRIBUTIONS}

All authors listed have made a substantial, direct, and intellectual contribution to the work and approved it for publication.

\section{ACKNOWLEDGMENTS}

DS is a post-doc recipient of a research grant salary in the frame of a research project (Tutor: GB) on "Pharmacoepidemiology of drugs used in the treatment of neuropsychiatric symptoms and pain in aged (over 65) people with dementia" funded by Calabria Region (POR Calabria FESR-FSE 2014/2020-Linea B) Azione 10.5.12. 


\section{REFERENCES}

Breivik, H., Collett, B., Ventafridda, V., Cohen, R., and Gallacher, D. (2006). Survey of chronic pain in Europe: prevalence, impact on daily life, and treatment. Eur. J. Pain 10 (4), 287. doi:10.1016/j.ejpain.2005.06.009

Diseases, G. B. D., and Injuries, C. (2020). Global burden of 369 diseases and injuries in 204 countries and territories, 1990-2019: a systematic analysis for the Global Burden of Disease Study 2019. Lancet. 396 (10258), 1204-1222. doi:10. 1016/S0140-6736(20)30925-9

Finnerup, N. B. (2013). Pain in patients with spinal cord injury. Pain. 154 (Suppl. 1), S71-s76. doi:10.1016/j.pain.2012.12.007

Karos, K., McParland, J. L., Bunzli, S., Devan, H., Hirsh, A., Kapos, F. P., et al. (2020). The social threats of COVID-19 for people with chronic pain. Pain. 161 (10), 2229-2235. doi:10.1097/j.pain.0000000000002004

Mills, S. E. E., Nicolson, K. P., and Smith, B. H. (2019). Chronic pain: a review of its epidemiology and associated factors in population-based studies. Br. J. Anaesth. 123 (2), e273-e283. doi:10.1016/j.bja.2019.03.023

Morrone, L. A., Rombolà, L., Pelle, C., Corasaniti, M. T., Zappettini, S., Paudice, P., et al. (2007). The essential oil of bergamot enhances the levels of amino acid neurotransmitters in the hippocampus of rat: implication of monoterpene hydrocarbons. Pharmacol. Res. 55 (4), 255-262. doi:10.1016/j.phrs.2006.11.010

Ossipov, M. H., Dussor, G. O., and Porreca, F. (2010). Central modulation of pain. J. Clin. Invest. 120 (11), 3779-3787. doi:10.1172/JCI43766

Reid, K. J., Harker, J., Bala, M. M., Truyers, C., Kellen, E., Bekkering, G. E., et al. (2011). Epidemiology of chronic non-cancer pain in Europe: narrative review of prevalence, pain treatments and pain impact. Curr. Med. Res. Opin. 27 (2), 449-462. doi:10.1185/03007995.2010.545813

Rombolà, L., Scuteri, D., Adornetto, A., Straface, M., Sakurada, T., Sakurada, S., et al. (2019). Anxiolytic-like effects of bergamot essential oil are insensitive to flumazenil in rats. Evidence-Based Complement. Altern. Med. 2019, 1. doi:10.1155/2019/2156873

Scuteri, D., Adornetto, A., Rombolà, L., Naturale, M. D., De Francesco, A. E., Esposito, S., et al. (2020a). Pattern of triptans use: a retrospective prescription study in Calabria, Italy. Neural Regen. Res. 15 (7), 1340-1343. doi:10.4103/1673-5374.272630

Scuteri, D., Adornetto, A., Rombolà, L., Naturale, M. D., Morrone, L. A., Bagetta, G., et al. (2019a). New trends in migraine pharmacology: targeting calcitonin gene-related peptide (CGRP) with monoclonal antibodies. Front. Pharmacol. 10, 363. doi:10.3389/fphar.2019.00363

Scuteri, D., Corasaniti, M. T., Tonin, P., and Bagetta, G. (2019b). Eptinezumab for the treatment of migraine. Drugs Today 55 (11), 695-703. doi:10.1358/dot.2019. 55.11.3069864

Scuteri, D., Crudo, M., Rombolà, L., Watanabe, C., Mizoguchi, H., Sakurada, S., et al. (2018a). Antinociceptive effect of inhalation of the essential oil of bergamot in mice. Fitoterapia 129, 20-24. doi:10.1016/j.fitote.2018.06.007
Scuteri, D., Garreffa, M. R., Esposito, S., Bagetta, G., Naturale, M. D., and Corasaniti, M. T. (2018b). Evidence for accuracy of pain assessment and painkillers utilization in neuropsychiatric symptoms of dementia in Calabria region, Italy. Neural Regen. Res. 13 (9), 1619-1621. doi:10.4103/1673-5374. 237125

Scuteri, D., Matamala-Gomez, M., Bottiroli, S., Corasaniti, M. T., De Icco, R., Bagetta, G., et al. (2020b). Pain assessment and treatment in dementia at the time of coronavirus disease COVID-19. Front. Neurol. 11, 890. doi:10.3389/ fneur.2020.00890

Scuteri, D., Piro, B., Morrone, L. A., Corasaniti, M. T., Vulnera, M., and Bagetta, G. (2017). The need for better access to pain treatment: learning from drug consumption trends in the USA. Funct. Neurol. 32 (4), 229-230. doi:10. 11138/fneur/2017.32.4.229

Scuteri, D., Rombolà, L., Morrone, L. A., Bagetta, G., Sakurada, S., Sakurada, T., et al. (2019c). Neuropharmacology of the neuropsychiatric symptoms of dementia and role of pain: essential oil of bergamot as a novel therapeutic approach. Int J Mol Sci. 20 (13), 3327. doi:10.3390/ijms20133327

Scuteri, D., Rombolá, L., Tridico, L., Mizoguchi, H., Watanabe, C., Sakurada, T., et al. (2019d). Neuropharmacological properties of the essential oil of bergamot for the clinical management of pain-related BPSDs. Curr Med Chem. 26 (20), 3764-3774. doi:10.2174/0929867325666180307115546

Scuteri, D., Vulnera, M., Piro, B., Bossio, R. B., Morrone, L. A., Sandrini, G., et al. (2021). Pattern of treatment of behavioural and psychological symptoms of dementia and pain: evidence on pharmacoutilization from a large real-world sample and from a centre for cognitive disturbances and dementia. Eur. J. Clin. Pharmacol. 77 (2), 241-249. doi:10.1007/s00228-020-02995-w

van Hecke, O., Torrance, N., and Smith, B. H. (2013). Chronic pain epidemiology and its clinical relevance. Br. J. Anaesth. 111 (1), 13-18. doi:10.1093/bja/ aet123

Yasko, J. R., and Mains, R. E. (2018). Chronic pain following spinal cord injury: current approaches to cellular and molecular mechanisms. Trends Cel Mol Biol. $13,67-84$.

Conflict of Interest: The authors declare that the research was conducted in the absence of any commercial or financial relationships that could be construed as a potential conflict of interest.

Copyright (c) 2021 Scuteri, Sakurada, Tonin, Corasaniti and Bagetta. This is an open-access article distributed under the terms of the Creative Commons Attribution License (CC BY). The use, distribution or reproduction in other forums is permitted, provided the original author(s) and the copyright owner(s) are credited and that the original publication in this journal is cited, in accordance with accepted academic practice. No use, distribution or reproduction is permitted which does not comply with these terms. 